\title{
Nanowire quantum dot surface engineering for high temperature single photon emission
}

\author{
Peng Yu ${ }^{1,2}$, Ziyuan $\mathrm{Li}^{2}$, Tongwei $W u^{1}$, Yitao Wang ${ }^{3}$, Xin Tong ${ }^{1}$, Chuanfeng ${ }^{3}{ }^{3}$, Zhongchang \\ Wang $^{4}$, Suhuai Wei ${ }^{5}$, Yunyan Zhang ${ }^{6}$, Huiyun Liu ${ }^{6}$, Lan Fu ${ }^{2}$, Yanning Zhang ${ }^{2}$, Jiang Wu ${ }^{1, *}$, Hark \\ Hoe Tan ${ }^{2}$, Chennupati Jagadish ${ }^{1,2}$, Zhiming Wang ${ }^{1, *}$ \\ ${ }^{1}$ Institute of Fundamental and Frontier Sciences, University of Electronic Science and \\ Technology of China, Chengdu 610054, P. R. China
}

${ }^{2}$ Department of Electronic Materials Engineering, Research School of Physics and Engineering, The Australian National University, Canberra, ACT 2601, Australia

${ }^{3}$ Key Laboratory of Quantum Information, University of Science and Technology of China, CAS, Hefei 230026, China

${ }^{4}$ Department of Quantum and Energy Materials, International Iberian Nanotechnology Laboratory (INL), Braga 4715-330, Portugal

${ }^{5}$ Beijing Computational Science Research Center, Beijing 100094, China

${ }^{6}$ Department of Electronic and Electrical Engineering, University College London, London WC1E 7JE, United Kingdom 
Abstract: Generating single photons at high temperature remains a major challenge, particularly for group III-As and III-P materials widely used in optical communication. Here, we report a high temperature single photon emitter based on a "surface-free" GaAs quantum dot in a GaAsP nanowire. By using self-catalyzed vapor-liquid-solid growth and simple surface engineering, we can significantly enhance the optical signal from the QDs with a highly polarized photoluminescence at $750 \mathrm{~nm}$. The "surface-free" nanowire quantum dots show photon antibunching up to $160 \mathrm{~K}$ and well resolved exciton lines as high as $220 \mathrm{~K}$.

KEYWORDS: single photon source, nanowire, quantum dot, photon antibunching, surface engineering

As a building block for quantum technology, single-photon emitters (SPEs) are critical parts in the realization of quantum communications, quantum information processing and quantum cryptography. Generating single photons can be achieved in a plethora of materials, such as color centers in crystals, ${ }^{1}$ two-dimensional materials, ${ }^{2}$ carbon nanotubes, ${ }^{3}$ and semiconductor quantum dots (QDs). ${ }^{4-8}$ The realization of single photon emission via an atom-like QD with discrete energy levels is a popular scheme because QDs have high emission rate, narrow emission linewidth and high fidelity anti-bunching. ${ }^{9,}{ }^{10}$ However, Stranski-Krastanov (S-K) grown QDs suffer from inhomogeneously broadened photon emission because of QD size fluctuations, local environmental variations with limited light extraction efficiency (1\%) and emission without polarization control. ${ }^{6,9-11}$ On the other hand, colloidal QDs exhibit blinking. ${ }^{12}$ Imbedding QDs in a photonic nanowire offers opportunities for obtaining SPEs with high light extraction efficiency, 
diminishing fine structural splitting, and polarization controllability. ${ }^{13,14}$ Compared with S-K QDs, imbedding QDs in nanowires is elegant where their position and size can be easily controlled, resulting in good spectral purity. The nanowires also act as a waveguide to provide the desired emission direction, ${ }^{13,15}$ with demonstrated external brightness in the $80 \%$ range. ${ }^{13,16}$ A positioncontrolled nanowire quantum dot (NWQD) demonstrates strongly entangled photon pairs with a fidelity as high as $0.859 \pm 0.006$ and concurrence of $0.80 \pm 0.02 .{ }^{14}$ To overcome the narrow bandwidth limitation of microcavities, tapered photonic nanowires have boosted light extraction efficiency of QDs to over 75\%. ${ }^{13,16}$ However, single photon emission of these QDs, S-K QDs or NWQDs, are sensitive to temperature, preventing their practical implementation. Recent literatures have reported QDs for single photon generation using II-VI, III-nitrides and conventional III-V materials. ${ }^{13,17-21} \mathrm{II}-\mathrm{V}$ and III-nitride SPEs are able to work at high temperature but at the short wavelength region that is less sensitive compared to conventional single-photon avalanche photodiodes. ${ }^{22}$ On the other hand, although mainstream (In,Ga)As materials emit single photons at the desired optical communication wavelengths, single photon emission is often limited at cryogenic temperature due to their relatively small exciton binding energies, QD-barrier band offsets, material imperfection and shallow electronic confinement in QDs. ${ }^{23-25}$ There are very few reports on SPEs using (In,Ga)As QDs operating above liquid nitrogen temperature and no report on (In, Ga)As NWQD SPEs that operates above liquid nitrogen temperature. . $^{9}$ 13, 26-29

Despite all the advantages of NWQDs, there are three main factors prevent III-As or III-P NWQD from emitting single photons at a high temperature. First, deep level traps induced by catalysts contamination during vapour-liquid-solid (VLS) growth of the nanowires may impair photon emission of NWQD ${ }^{30-32}$. Second, it is difficult to fabricate ideal disk-like QD in a nanowire 
with sharp interfaces and crystal imperfections. Stacking faults, microtwins and phase polytypism are usually formed during growth, which act as scattering centres or charge traps. ${ }^{19,30,33}$ Rapid photoluminescence (PL) thermal quenching has been observed in nanowires and QDs due to these defects and charge traps. ${ }^{30,34}$ Last but not least, surface states also degrade the optical properties of the NWQD through nonradiative recombination as temperature increases and has been a major concern in many optoelectronic applications. ${ }^{35}$

Here we present a promising SPE based on defect-free self-catalyzed GaAsP/GaAs NWQDs grown by molecular beam epitaxy (MBE). After removing the surface states, the SPE operates above liquid nitrogen temperature and at a desired wavelength of $750 \mathrm{~nm}$. In order to achieve higher photon antibunching, impurity and defect incorporation have to be minimized in addition the surface engineering of the NWQDs. A variety of surface state treatment methods, including dry and wet treatment, have been already been investigated to remove the surface states of lowdimensional materials. ${ }^{36,37}$ However, surface states removal by chemical or physical deposition usually requires precise control of temperature and thickness. Surface engineering techniques based on aqueous solutions lead to some amount of residual oxides and may impair the structural quality of the surface. Here, we use alcoholic ammonium sulfide (AS) solution, which can provide a more effective passivation and long-term stability in ambient. After removing the surface states, the $\mu$-PL of the NWQD is significantly enhanced up to $220 \mathrm{~K}$ and the NWQDs show photon antibunching at $160 \mathrm{~K}$ with a $\mathrm{g}^{2}(\tau=0)$ value of 0.482 .

\section{Methods/Experimental NWQD growth}

The heterojunction GaAsP/GaAs NWQDs were grown by MBE following the methods described in Ref. ${ }^{30}$. Briefly, GaAsP nanowires were grown by Ga-assisted VLS process and a 
GaAs QD region was formed in the middle of GaAsP wires by switching off the supply of phosphorus. In order to avoid any instability of the self-catalysed nanowire growth, As beam pressure was increased to compensate the reduced supply of phosphorus during QD growth. After the QD growth, the equivalent beam pressure of As and P molecules were restored to the initial conditions without any interruption.

\section{Electron microscopy characterization}

The morphology and crystal structure of NWQDs were characterized by scanning electron microscopy (SEM) equipped with a Gatan MonoCL4 Elite cathodoluminescence (CL) system (FEI Verios) operated at $5 \mathrm{kV}$, and transmission electron microscopy (TEM) equipped with an energydispersive X-ray (EDX) spectroscopy (JEOL 2100F), respectively. NWQDs were mechanically transferred on a lacey carbon copper grid for TEM characterization. The high-resolution angular dark field (HAADF) images were obtained with a 200-kV scanning TEM (STEM, JEM-2100F, JEOL). (Supporting Information Figure S1)

\section{FDTD simulation and density functional theory calculations}

Finite-Difference Time-Domain (FDTD) simulations were performed using a commercial software, Lumerical Solutions. The modeled structure consists of a GaAsP nanowire (with various diameter at a fixed length of $2500 \mathrm{~nm}$ ) and an electric dipole emitter which is used to represent the GaAs QD in the GaAsP nanowire. Spin-polarized density functional theory (DFT) calculations were performed using the plane wave-based Vienna ab initio simulation package (VASP); The surface of the GaAs QD is assumed to have a six-fold symmetric structure with six $\{110\}$ facets linked by $\{112\}$ facets. (Supporting Information Figure S2 and DFT calculation)

\section{Surface treatment}

The transferred NWQDs were firstly immersed in $35 \% \mathrm{NH}_{4} \mathrm{OH}$ for 2 min to remove the surface oxide layer, followed by DI water rinse and $\mathrm{N}_{2}$ blow dry. The samples were immediately immersed 
into $20 \%$ aqueous $\mathrm{AS}\left(\left(\mathrm{NH}_{4}\right)_{2} \mathrm{~S}_{\mathrm{x}}\right)$ solution, diluted by 2-propanol $\left(2-\mathrm{C}_{3} \mathrm{H}_{7} \mathrm{OH}, \mathrm{IPA}\right)$ in the ratio of 1:5, for 10 min at room temperature, followed by IPA rinse and blow dry with $\mathrm{N}_{2}$ gas.

\section{Optical characterisations of NWQDs}

Individual nanowires were transferred to marked silicon substrates with $250 \mathrm{~nm} \mathrm{SiO}_{2}$ layer grown by plasma enhanced chemical vapour deposition. Samples were placed onto a helium flow cryostat for low-temperature measurements. A time-correlated single photon counting system was used for time-resolved PL (TRPL) measurements with an instrument response of $\sim 50$ ps at fullwidth-at-half-maximum (FWHM). Second order correlation measurements were carried out under CW optical excitation using a Hanbury Brown and Twiss (HBT) interferometer setup with two nominally identical silicon avalanche photodiodes and a 50/50 beam splitter. Nanowires were pumped using a frequency-doubled solid-state laser (femto-TRAIN IC-Yb-2000, $\lambda=522 \mathrm{~nm}$, repetition rate $20.8 \mathrm{MHz}$, pulse length $400 \mathrm{fs}$ ) through a $60 \times / 0.70$ numerical aperture objective lens (NIKON S PLAN FLUOR). The emission from single NWQDs was collected through the same objective lens and spectrally filtered to eliminate signal from the pump laser.

Results/Discussion The NWQDs were grown on a $\mathrm{Si}$ (111) substrate, as shown in figure 1(a). The freestanding NWQDs have diameters and lengths ranging from $\sim 30-60 \mathrm{~nm}$ and $\sim 2-5 \mu \mathrm{m}$, respectively, as shown in figure 1(b). According to the SEM image, no kinking is observed in the GaAsP nanowires even with a GaAs QD segment inserted in the middle of the nanowire, indicating that the surface interfacial energetics has been carefully controlled during growth (Figure S4). 

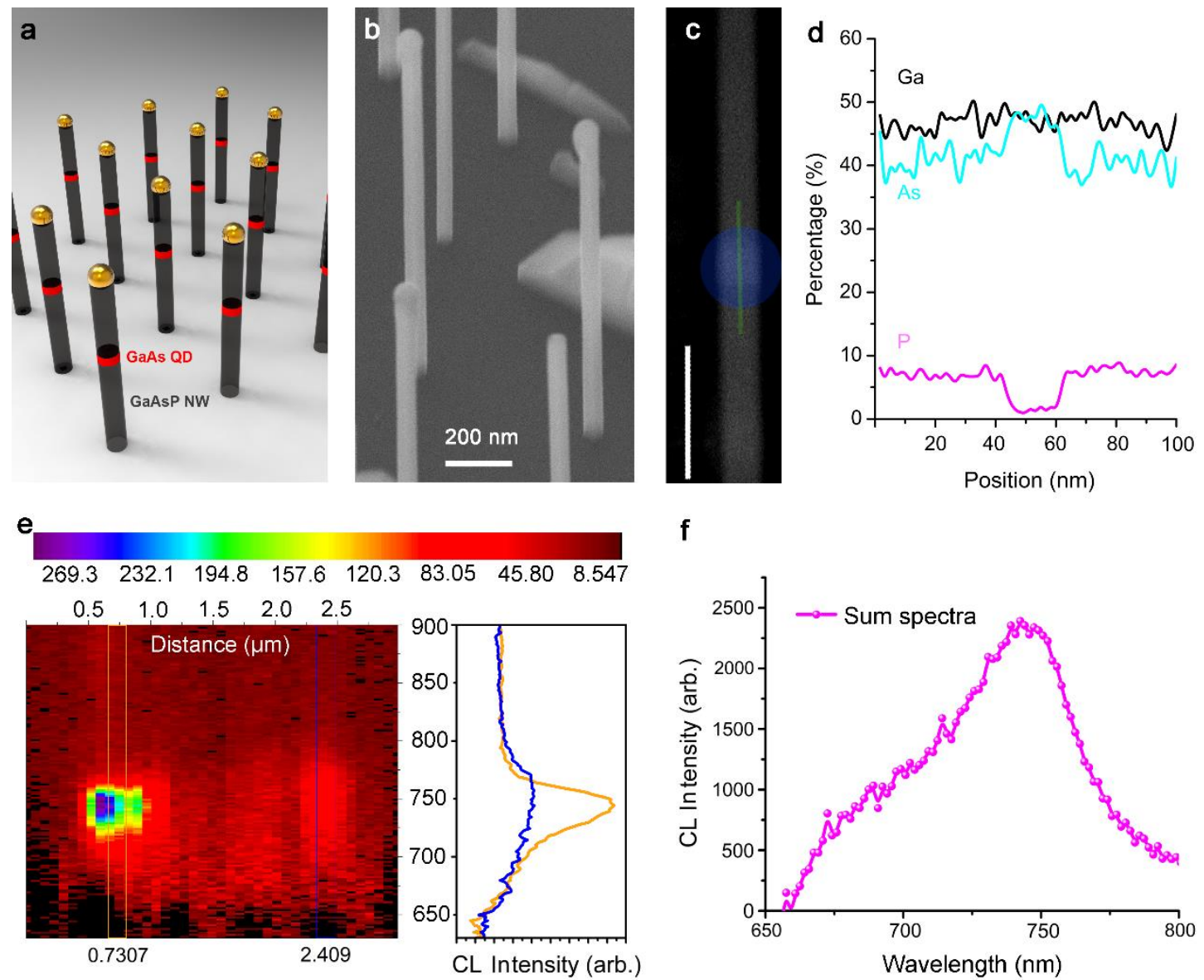

Figure 1. Structural and spectral information of the GaAsP/GaAs NWQDs. a. Schematic of the self-catalyzed GaAsP/GaAs NWQDs grown by the VLS mechanism on Si (111) substrates. b SEM images of the GaAsP/GaAs NWQD with a tilt angle of $30^{\circ}$; the scale bar is $200 \mathrm{~nm}$. c. Low-magnification TEM image of a GaAs QD imbedded in a GaAsP nanowire; the scale bar is $100 \mathrm{~nm}$. d. Elemental atomic percentage profile taken along the QD area region as indicated by the green line in (c). e. 2D mapping of CL intensity of an individual NWQD after surface state passivation with attached CL spectra taken from the region marked yellow (GaAs QD) and blue in the color map. f. Total CL spectra taken across the NWQD.

Low-magnification TEM image confirms that the GaAsP nanowire contains a single GaAs QD with a width of $\sim 30 \mathrm{~nm}$ and height of $15 \mathrm{~nm}$, as shown in figure 1(c). The HAADF images 
confirms the zinc-blend structure of the GaAs QD and the absence of stacking faults, microtwins and phase polytypism along the nanowire, as shown in Figure S1 (a)-(d). The interface of the QD is intrinsically sharp down to atomic level with precise control of geometry and composition while their self-assembled QD counterparts have intermixed interfaces (Figure S1 (e) and (f)).$^{38}$ The elemental composition of the GaAsP/GaAs NWQD is analyzed by EDX, the results of which are plotted in figure 1(d). The atomic percentage profile demonstrates comparable Ga and As (50\%) percentages with small $\mathrm{P}$ in the GaAs QD region. A small amount of $\mathrm{P}$ in $\mathrm{QD}$ region can be attributed to the residual $\mathrm{P}$ source in the chamber after switching off the $\mathrm{P}$ source.

Surface states that appear above or below bandgap are detrimental to the band-edge emission, leading to a reduced brightness and broadening of FWHM. ${ }^{39}$ Proper removal of the surface states and traps in QDs can eliminate or prevent non-radiative decay pathways, recovering band-edge emission and thus improve colour purity and brightness of QDs. The surface treatment procedure removes the native oxide and forms a thin chemisorbed film of sulfur, as shown in figure 2(a). Before surface treatment, no signal can be detected from CL measurement at room temperature but strong emission from the QD region can be seen after AS treatment, as shown in figure 1(e) and (f), meaning that it has a possibility operating at room temperature as a SPE via electrically driven pump. Success removal of NWQD surface states is also confirmed by PL spectra (assemble NWQD and single GaAsP nanowire) and room-temperature Raman spectra (assemble NWQD) (Figure S5 and S6). The $\mu$-PL of single GaAsP nanowires (same grown method as the NWQDs but without the GaAs QD) demonstrates five orders of magnitude PL enhancement after passivation. The Raman signal from the QD is weak and thus we only focus on the Raman signal from the GaAsP wire to evaluate the effectiveness of the surface state removal process. The peak marked $\mathrm{L}^{-}\left(\sim 268.9 \mathrm{~cm}^{-1}\right)$ in figure $\mathbf{S 5}(\mathbf{c})$ is caused by scattering from coupled phonon-plasmon 
modes in the bulk while the peak marked LO $\left(\sim 284.8 \mathrm{~cm}^{-1}\right)$ originates from the surface depletion layer. As GaAsP/GaAs NWQDs react with $\left(\mathrm{NH}_{4}\right)_{2} \mathrm{~S}$, the LO peak intensity decreases, meaning a large reduction in surface band bending, as observed in Ref. ${ }^{40}$.

The effect of surface engineering of the nanowires is theoretically calculated by DFT method. The calculation is based on clean surfaces that takes reconstruction into account with an Astermination surface. There are two possible adsorption sites, S1 and S2, of sulfur atoms, as shown in figure 2(b). The calculation results show that a sulfur atom prefers to be adsorbed on S1 site between $\mathrm{As}$ and $\mathrm{Ga}$ atoms in figure 2(c), with an adsorption energy ( $\left.\mathrm{E}_{\mathrm{adS}}\right)$ of $-0.112 \mathrm{eV}$. Subsequently, we calculated the adsorption energy of the surface with various oxygen density in Figure 2(d) and S3, in which only the surface structure in figure 2(d) has adsorption site that the sulfur atom prefers, with $\mathrm{E}_{\mathrm{adO}}=-1.422 \mathrm{eV}$. It suggests that the sulfur may partially adsorbed at oxidized surface in figure 2(d). The calculation results show that sulfur adsorption energy sharply increases to $-1.265 \mathrm{eV}$ in as shown in figure 2(e). We further calculated the density of states (DOS) of the structure, as shown in figure 2(f). The reconstructed clean surface (Clean) shows a bandgap of $\sim 0.57 \mathrm{eV}$, and then reduces to zero since GaAs surface is oxidized (Ot). Finally, the bandgap is restored to $0.27 \mathrm{eV}$ as the oxidized surface is partly sulfurised $(\mathrm{St})$. The effect of passivation also experimentally confirmed by TRPL, as a longer carrier lifetime shown in figure 2(g). Due to large surface area-to-volume ratios, GaAs QDs or nanowire exhibit a high surface recombination with short carrier lifetimes of only a few picoseconds. ${ }^{41}$ At $10 \mathrm{~K}$, the minority carrier lifetime, $\tau_{\mathrm{mc}}$, is increased from 0.167 to $0.346 \mathrm{~ns}$ after passivation, indicating bandgap recovery. 


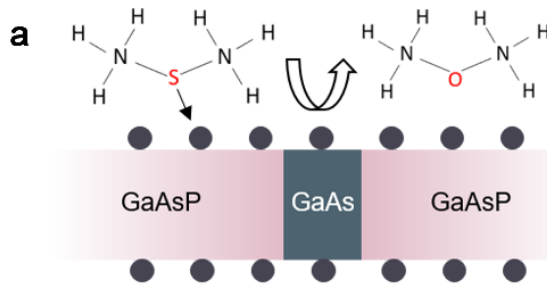

b

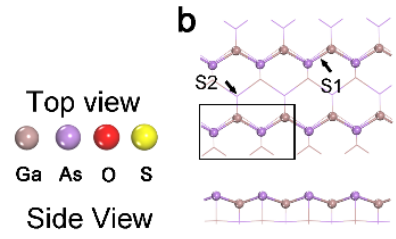

Clean surface c s
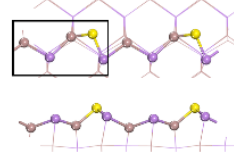
Sulfide surface $0.5 \mathrm{ML} \quad \mathrm{E}_{\mathrm{ads}}=-0.112 \mathrm{eV}$

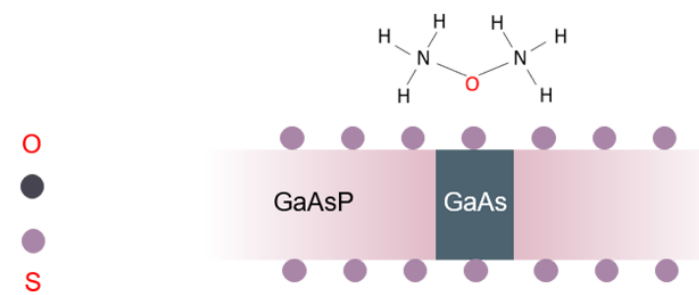

d

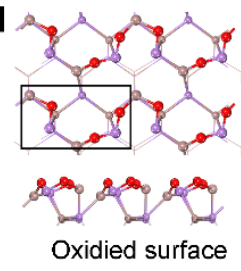

$1.5 \mathrm{ML} \quad \mathrm{E}_{\text {ado }}=-1.422 \mathrm{eV}$ e

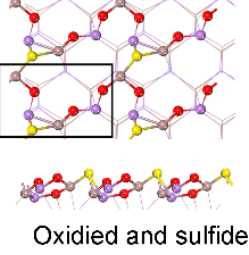

$2 \mathrm{ML} \quad \mathrm{E}_{\mathrm{ads}}=-1.265 \mathrm{eV}$

$\begin{array}{llllllllll}\text { f } & -5 & -4 & -3 & -2 & -1 & 0 & 1 & 2 & 3\end{array}$
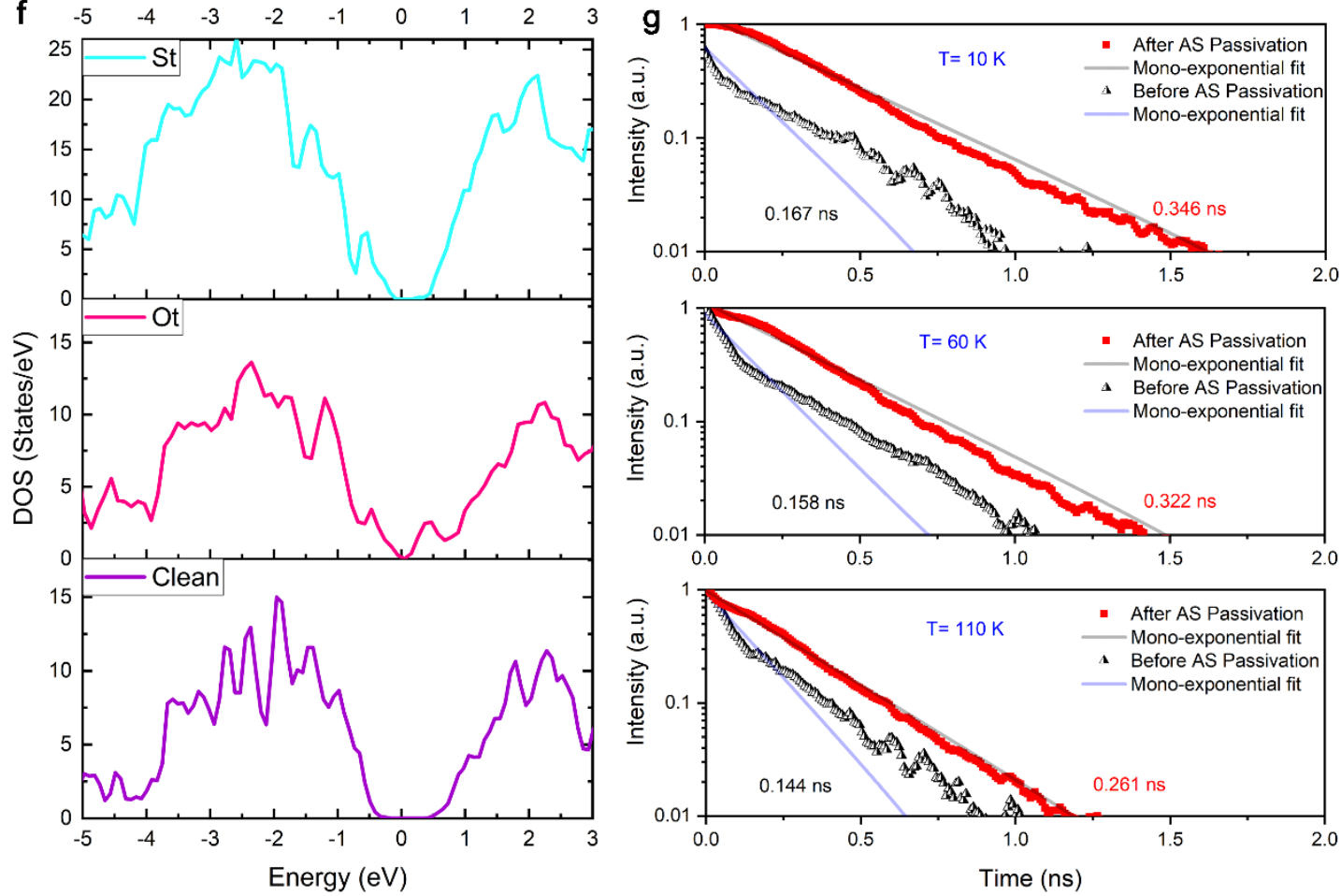

Figure 2. The effect of surface engineering of the GaAsP/GaAs NWQD. a. Schematic of the processes happening at the NWQD during surface treatment of NWQD. b-e. Schematic of GaAs QD surface construction in DFT calculation with rectangle unit cell shown. S1 and S2 indicate two adsorption sites on GaAs surface; ML is adsorption coverage of atoms. $\mathbf{f}$ DOS of the clean, oxygen reconstructed and sulfur (partially) reconstructed GaAs (110) surface. g. TRPL at 
emission wavelength from the NWQD before and after AS treatment at 10, 60 and $110 \mathrm{~K}$. Monoexponential fits with $\tau_{\mathrm{mc}}$ are shown for each decay.

Figure 3(a) and (b) shows the NWQD after surface treatment and its PL spectra with increasing excitation power from $52 \mathrm{nW}$ to $11.79 \mu \mathrm{W}$, respectively. The tapered nanowire can boost the light extraction efficiency above $80 \%$ due to minimized total internal reflection at the semiconductorair interface by tapering the nanowire tip while a nanowire quantum dot without tapering demonstrated an extraction efficiency of $\sim 17 \%{ }^{42}$ Figure 3(c) plots the integrated intensities of $I(X)$ and $I(X X)$ which are equal to each other at saturation power. The integrated intensity decreases with increasing pump energy, which can be explained by the transition of electrons among the dark exciton, bright exciton, and biexciton. ${ }^{43}$ The spectra present a narrow excitonic line $(X)$ with slope of $\sim 1.0$ peaks at $\sim 1.638 \mathrm{eV}$ and bi-excitonic line $(X X)$ with slope of $\sim 1.88$ peaks at $\sim 1.636$ $\mathrm{eV}$, respectively. The reduced emission intensity at higher excitation power may be ascribed to temperature effect of PL electrons and scattering to wire by phonon scattering or between electrons.

For quantum information applications, control of polarization is essential. Polarizationdependent PL spectra are obtained from the GaAsP/GaAs NWQD dispersed on the substrate by placing a linear polarizer in front of the monochromator. The measured integrated intensity of the $X$ line emission from the NWQD as a function of angle is plotted in figure 3(d) and (e). The experiment data are fitted to equation (1):

$$
I(\theta)=a+b \cos ^{2}\left(\theta-\theta_{0}\right)
$$

where $\theta_{0}$ is the linear polarization direction of the QD line, $a$ and $a+b$ are minimum and maximum values of the integrated PL intensity along the growth direction (define as $y$ ) of GaAsP/GaAs NWQD. The degree of polarization, $\rho$, is characterized by equation (2): 


$$
\rho=\left(I_{\|}-I_{\perp}\right) /\left(I_{\|}-I_{\perp}\right)
$$

where $I_{/ /}$and $I_{\perp}$ is the light intensity parallel and perpendicular to the $y$ direction, respectively. The $\rho$ of NWQD is $20.3 \%$ (before surface treatment) and $82.5 \%$ (after surface treatment). Due to the large refractive index contrast between the nanowire and the surrounding air, the NWQD demonstrates a strong linear polarization along the growth direction, as shown in figure $\mathbf{S 7} .{ }^{44}$ The surface treatment has a great impact on the $y$ direction but with only a minor effect on other directions since the PL intensity changes only slightly after surface states removal when light perpendicular to the nanowire axis, as shown in figure 3(e). This again justifies the success removal of surface states on NWQD. 
a
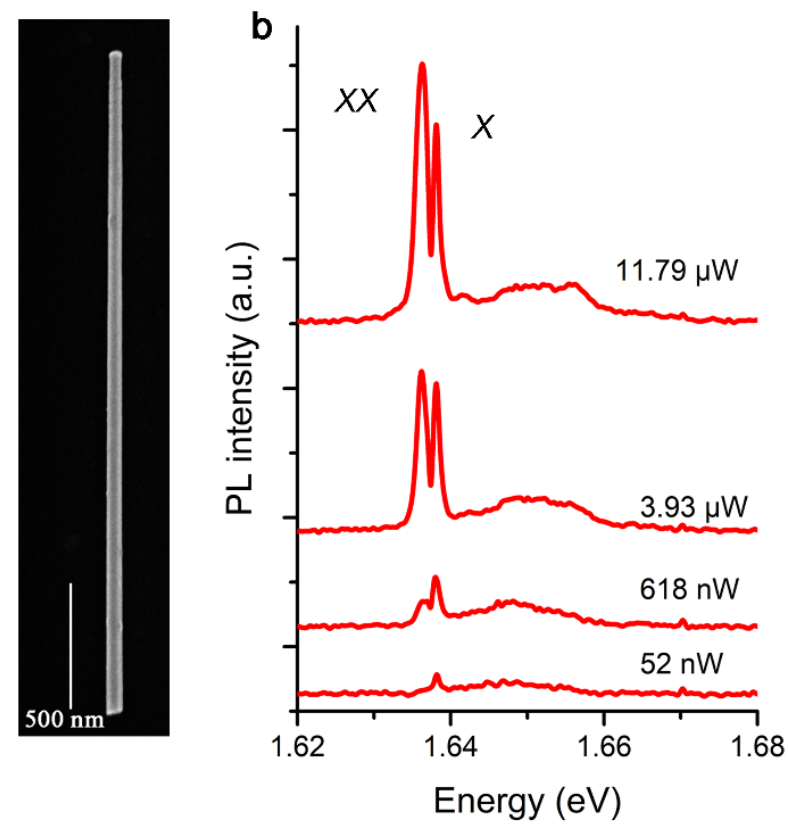

d

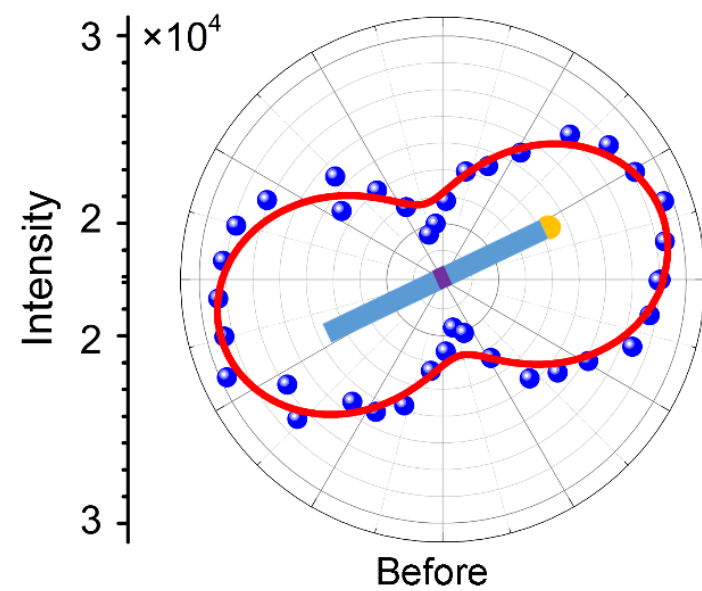

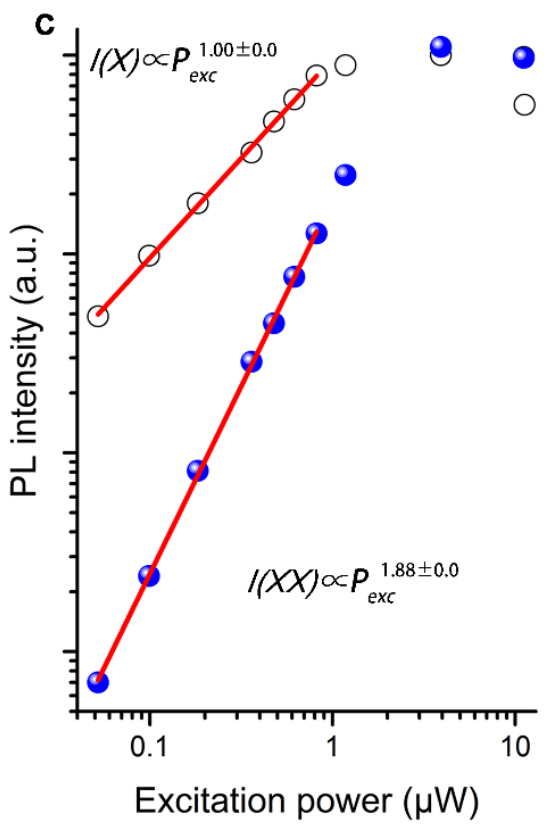

e

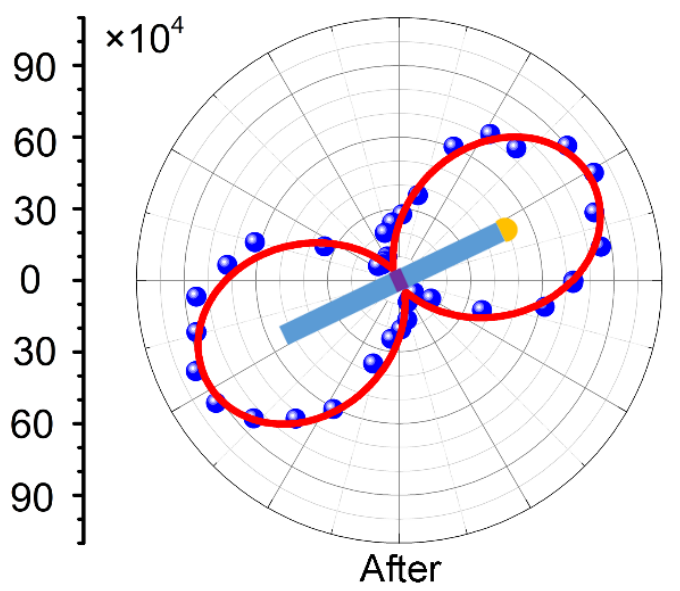

Figure 3. Power and polarization-dependent relationship of a single GaAsP/GaAs NWQD. a. SEM image of a NWQD lying horizontally on a $\mathrm{SiO}_{2}$ substrate. b. Power-dependent $\mu$-PL of a single NWQD measured at $10 \mathrm{~K}$ with increasing optical pump power. c. Integrated counts of $X$ and $X X$ peaks as a function of excitation power. Polarization-dependent analysis of the emission from the NWQD (d) before and after (e) AS treatment, measured at $10 \mathrm{~K}$ with an excitation power of $\sim 3.6 \mu \mathrm{W}$. The nanowire orientation is schematically shown in the polar axis. 
The $\mu$-PL spectra measured from a single device before surface state removal at 10 and $60 \mathrm{~K}$ are shown in figure 4(a). At $10 \mathrm{~K}$ the QD exhibits a sharp emission at $1.638 \mathrm{eV}$, with a FWHM of 1.2 meV. At $60 \mathrm{~K}$, the luminescence redshifts to $1.366 \mathrm{eV}$ with a FWHM of $1.8 \mathrm{meV}$. As the temperature increases further, no PL signal can be observed. The rapid PL quenching of NWQD results from surface states presented in the GaAsP barrier near the NWQD. ${ }^{30}$ To confirm single photon emission of $X$ line, we performed the second-order correlation function, $\mathrm{g}^{2}(\tau)$, of $X$ emission with an exciton power close to saturation, as shown in figure $4(\mathbf{b})$. The $\mathrm{g}^{2}(\tau)$ indicates a multiphoton suppression evident by a pronounced dip in the autocorrelation signal. If a measurement $\mathrm{g}^{2}(\tau=0)<0.5$, it suggests the material can be used as a single photon source. The measured data can be fitted with the function equation (3),

$$
g^{2}(\tau)=1-A \times \exp \left(-|\tau| / \tau_{r}\right)
$$

where $1-A$ is $g^{2}(\tau=0)$. The corrected $g^{2}(\tau=0)$ of NWQD before surface treatment is 0.16 and 0.21 at 10 and $60 \mathrm{~K}$, respectively. At higher temperature, the exciton linewidth broaden to $\sim 1.8 \mathrm{meV}$, which leads to a degradation of $\mathrm{g}^{2}(\tau=0)$ value and is usually attributed to spectral contamination from thermally broadened excited states. ${ }^{20}$ It is worth noting that the finite value of the secondorder correlation at zero time delay may be ascribed to background signal or limited time resolution of the measurement system. 

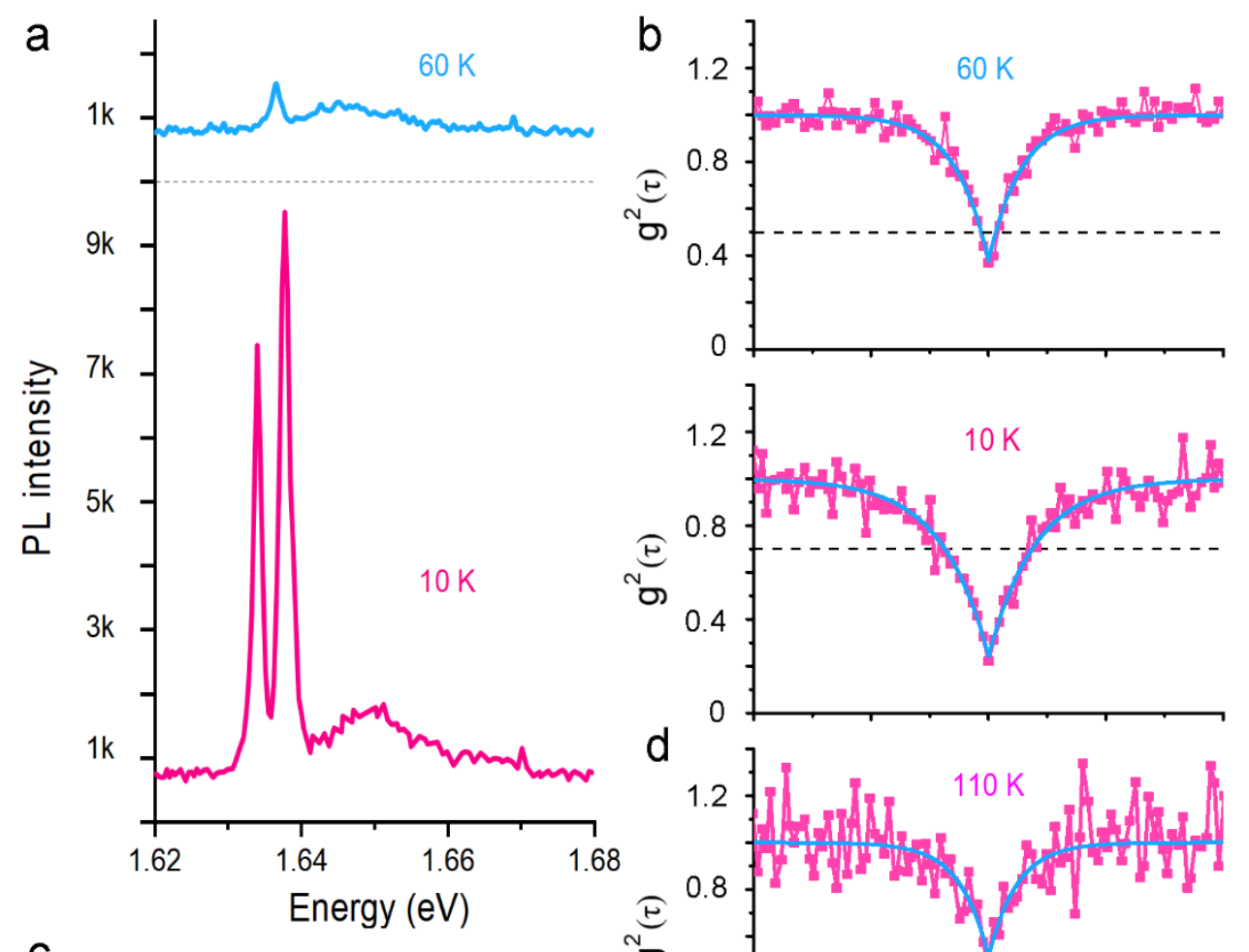

C
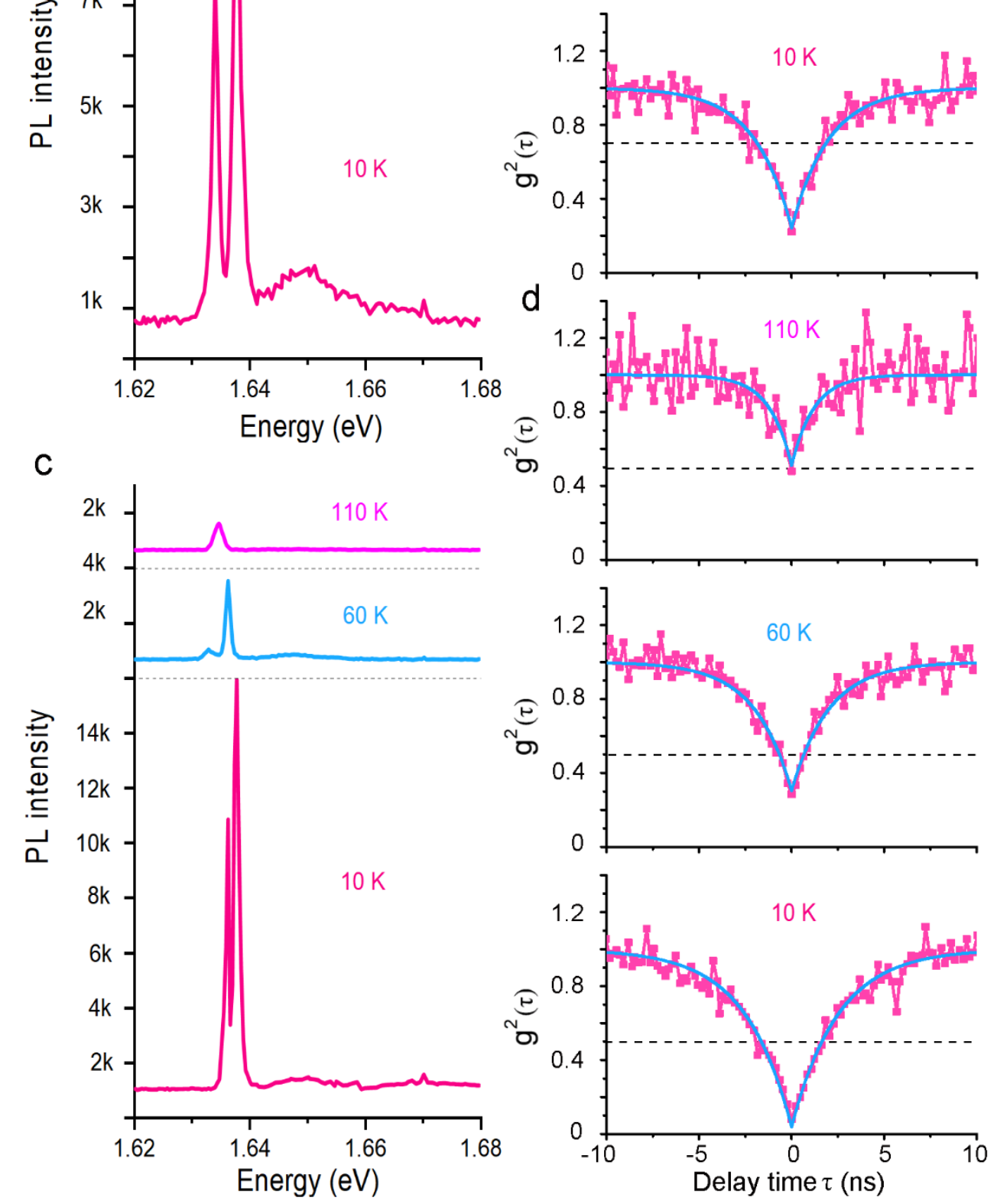

Figure 4. Single photon emission at different temperature. a. $\mu$-PL spectra of a typical single NWQD measured at 10 and $60 \mathrm{~K}$; the excitation power is $819 \mathrm{nW}, 2.46 \mu \mathrm{W}$ respectively. b.

Second-order correlation function, $\mathrm{g}^{2}(\tau)$, of the $X$ peak at 60 and 10 K. c. $\mu$-PL spectra of a single 
NWQD measured at 10, 60 and $110 \mathrm{~K}$, respectively; the excitation power is $819 \mathrm{nW}, 2.46 \mu \mathrm{W}$ and $7.38 \mu \mathrm{W}$. d. Second-order correlation function, $\mathrm{g}^{2}(\tau)$, of the $X$ line peak at 110,60 and $10 \mathrm{~K}$ respectively.

The surface engineering leads to a significantly increased PL signal of the single NWQD. The exciton spectrum is measured up to $110 \mathrm{~K}$ with a FWHM of $2.0 \mathrm{meV}$, as shown in figure 4(c). The lifetime, measured $\mathrm{g}^{2}(\tau=0)$, FWHM are compared before and after surface treatment in table S1. Before surface engineering, non-radiative recombination serves as pathway for carriers while only band-edge emission is occurred after surface state removal, as illustrated in figure $\mathbf{S 8}$. The PL efficiency can be defined as $\mathrm{I}_{\mathrm{r}} /\left(\mathrm{I}_{\mathrm{r}}+\mathrm{I}_{\mathrm{nr}}\right)$, where $\mathrm{I}_{\mathrm{r}}$ and $\mathrm{I}_{\mathrm{nr}}$ are radiative and nonradiative transition probabilities, respectively. Nonradiative recombination becomes more significant at higher temperatures. If trap states introduced by crystalline defects, surface states and metallic catalyst are removed, then PL efficiency can be increased. It is worth noting that the maximum surface states density is located near the band-edge but the Fermi energy is pinned above the conduction band. ${ }^{39}$ Therefore, some above-band-edge surface state still remain and this is consistent with measured minority carrier lifetime of $\sim 0.35$ ns while the "surface free" GaAs is $\sim 1 \mathrm{~ns} .{ }^{41}$

The increased PL intensity and narrowed FWHM makes single photon emission from the NWQDs with surface engineering less sensitive to temperature, as shown in figure $4(d)$. The corrected $\mathrm{g}^{2}(\tau=0)$ values are $0.05,0.19$ and 0.32 under 10,60 and $110 \mathrm{~K}$, respectively. The observation of photon antibunching at elevated temperature confirms the effectiveness of the surface treatment. Since thermal quenching is reduced, PL from a single NWQD can be measured up to $220 \mathrm{~K}$ with a corrected $\mathrm{g}^{2}(\tau=0)$ of 0.482 at $160 \mathrm{~K}$, as plotted in figure $\mathbf{5 ( a )}$ and figure $\mathbf{S 9}$. At 
$220 \mathrm{~K}$, the non-Lorentzian sidebands can be attributed to acoustic-phonon related sideband of GaAs QD emission. ${ }^{20}$ Degradation of $\mathrm{g}^{2}(\tau=0)$ as temperature increases has been extensively reported in many nonsite-controlled QD system. ${ }^{20}$ The NWQD without surface states removal show no detectable signal after $220 \mathrm{~K}$ and hence $\tau_{\mathrm{mc}}$ could not be measured. However, for the passivated NWQD $\tau_{\mathrm{mc}}$ could be obtained up to $300^{\circ} \mathrm{C}$ (figure S10), suggesting that our GaAsP/GaAs NWQD may have potential for room temperature application. There are two possible factors influence the NWQD operating above $160 \mathrm{~K}$. First, the pumping power need to be increased due to the decreased PL efficiency at high temperature. This leads to carrier transfers to the GaAsP wire and thus degrades the second-order intensity correlation. ${ }^{9}$ Second, as temperature increases, optical phonon scattering become prominent. Future investigation should quantitatively investigate the influence of each factors on high temperature single photon emission. To compare our results with those already reported in the literature, we plot (operating) temperature vs $g^{2}(\tau=0)$ for some of the III-V QDs for single photon emission, as shown in figure 5(b). At low temperature, there is a clear dip at time delay zero, meaning anti-bunching in the photon statistics, while at 160 $\mathrm{K}$, an obvious dip still present with $\mathrm{g}^{2}(\tau=0)<0.5$ which can be seen as a non-classical quantum source. 


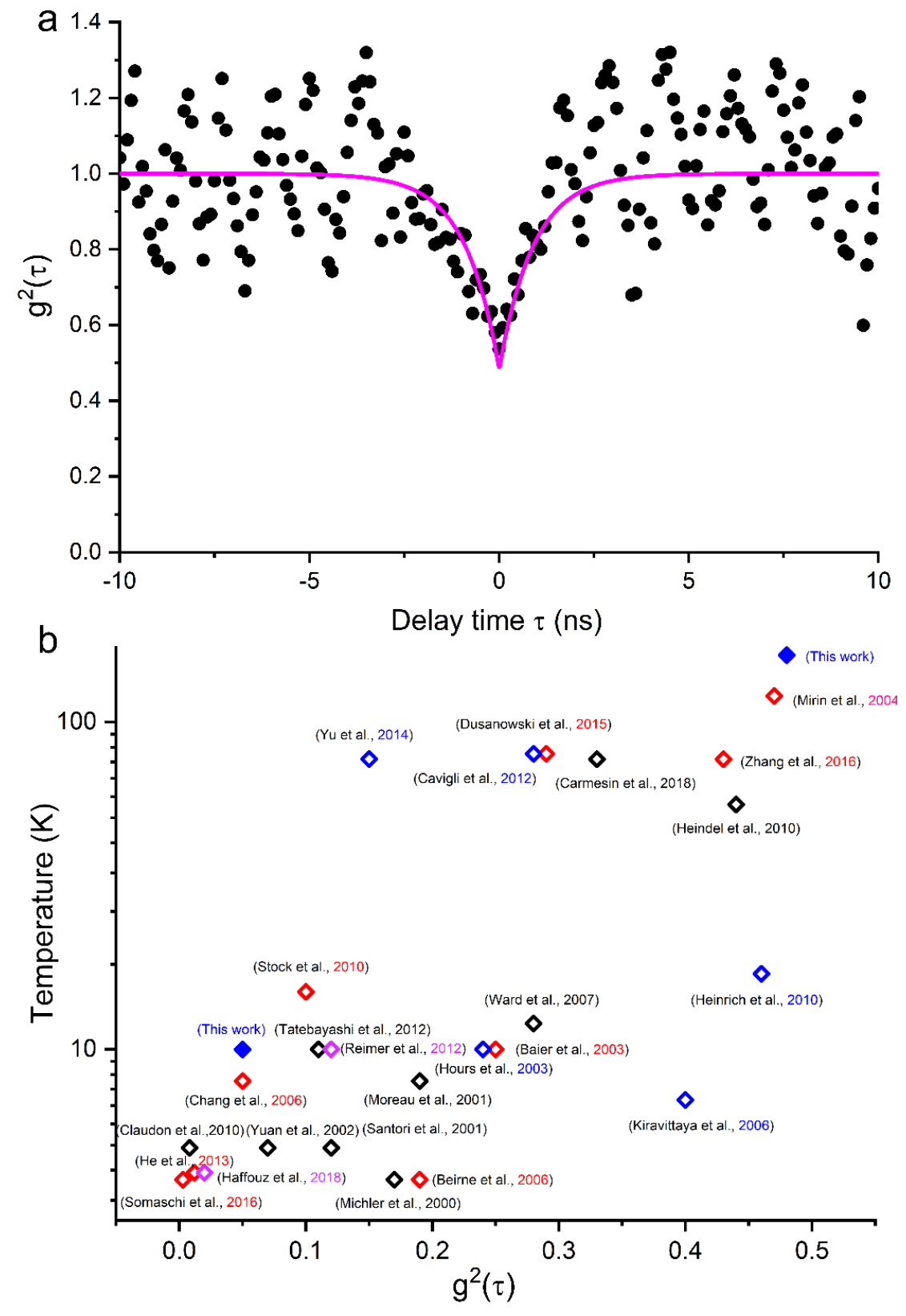

Figure 5. Comparison of $\mathrm{g}^{2}(\tau=0)$. a. Corrected second-order correlation function of the single GaAsP/GaAs NWQD at 160 K. b. Comparison of single photon emitters based on reported III-V QDs operating at different temperature; blue GaAs, purple InAsP, red InGaAs, black InAs QDs. References in figure 5(b) are listed in Supporting Information. 


\section{Conclusions}

In conclusion, we have fabricated GaAsP/GaAs NWQDs and obtained high temperature single photon emission by surface engineering of the NWQDs. Before removing surface states of the NWQDs, they show a rapid PL quenching. Second-order autocorrelation measurements were carried out at the exciton energy, showing photon antibunching only up to $60 \mathrm{~K}$ with a background corrected $\mathrm{g}^{2}(\tau=0)$ of 0.21 for the NWQDs without engineering. Surface states were removed by alcoholic ammonium sulfide solution treatment to recover band edge emission from the QDs, after which, the PL intensity and FWHM of the NWQD were significantly improved, and as a result a corrected $\mathrm{g}^{2}(\tau=0)$ of 0.48 was measured at a temperature as high as $160 \mathrm{~K}$. The surface treatment also boosts the linear degree of polarization of the NWQD to $82.5 \%$ from $20.3 \%$. The surface treatment is particularly promising for obtaining single photon sources operating at roomtemperature and their applications, for example, room-temperature quantum communication.

\section{Supporting Information}

STEM image of NWQD; FDTD and DFT Simulation; Low-magnification SEM images of NWQD; GaAsP/GaAs NWQD with enhanced optical properties after passivation, including PL and Raman spectra; output polarization; schematics of the recombination process; light emission at a high temperature; possibility operating at room temperature; table S1.

\section{AUTHOR INFORMATION}

\section{Corresponding Author}

*E-mail: Jiangwu@uestc.edu.cn

*E-mail: zhmwang@uestc.edu.cn 


\section{Author Contributions}

J.W., Z.M.W. and H.Y.L. conceived and designed this research. P.Y., Z.Y.L., Y.T.W., X.T.,

C.F.L., Z.C.W. and Y.Y.Z performed the experiments, J.W., L.F, H.T. and C.J. analysed the data. P.Y., T.W.W, S.H.W and Y.N.Z conducted the DFT and FDTD simulations. P.Y. and J.W. wrote the paper.

\section{Funding Sources}

The work is supported by EPSRC Grant No. EP/P000886/1 and EPSRC National Epitaxy Facility, National Basic Research Program of China (Grant No. 2013CB933301), and the Australian Research Council. We acknowledge the support from the ANFF ACT Node in carrying out this research.

\section{ACKNOWLEDGMENT}

We thank Dr. Mark Lockrey for the CL measurement. The authors declare no competing interests.

\section{REFERENCES}

1. Mizuochi, N.; Makino, T.; Kato, H.; Takeuchi, D.; Ogura, M.; Okushi, H.; Nothaft, M.; Neumann, P.; Gali, A.; Jelezko, F., Electrically driven single-photon source at room temperature in diamond. Nat. Photonics 2012, 6 (5), 299.

2. He, Y.-M.; Clark, G.; Schaibley, J. R.; He, Y.; Chen, M.-C.; Wei, Y.-J.; Ding, X.; Zhang, Q.; Yao, W.; Xu, X., Single quantum emitters in monolayer semiconductors. Nat. Nanotechnol. 2015, 10 (6), 497-502.

3. Jeantet, A.; Chassagneux, Y.; Raynaud, C.; Roussignol, P.; Lauret, J.-S.; Besga, B.; Estève, J.; Reichel, J.; Voisin, C., Widely tunable single-photon source from a carbon nanotube in the Purcell regime. Phys. Rev. Lett. 2016, 116 (24), 247402.

4. $\quad$ Michler, P.; Kiraz, A.; Becher, C.; Schoenfeld, W. V.; Petroff, P. M.; Zhang, L.; Hu, E.; Imamoglu, A., A Quantum Dot Single-Photon Turnstile Device. Science 2000, 290 (5500), 22822285.

5. Sonia, B.; Kelley, R.; Jelena, V., Engineered quantum dot single-photon sources. Rep. Prog. Phys. 2012, 75 (12), 126503. 
6. Aharonovich, I.; Englund, D.; Toth, M., Solid-state single-photon emitters. Nat. Photonics 2016, 10 (10), 631-641.

7. Kako, S.; Santori, C.; Hoshino, K.; Götzinger, S.; Yamamoto, Y.; Arakawa, Y., A gallium nitride single-photon source operating at 200 K. Nat. Mater. 2006, 5, 887.

8. Yuan, Z.; Kardynal, B. E.; Stevenson, R. M.; Shields, A. J.; Lobo, C. J.; Cooper, K.; Beattie, N. S.; Ritchie, D. A.; Pepper, M., Electrically Driven Single-Photon Source. Science 2002, 295 (5552), 102-105.

9. Mirin, R. P., Photon antibunching at high temperature from a single InGaAs/GaAs quantum dot. Appl. Phys. Lett. 2004, 84 (8), 1260-1262.

10. Sebald, K.; Michler, P.; Passow, T.; Hommel, D.; Bacher, G.; Forchel, A., Single-photon emission of CdSe quantum dots at temperatures up to 200 K. Appl. Phys. Lett. 2002, 81 (16), 29202922.

11. Davanço, M.; Rakher, M. T.; Wegscheider, W.; Schuh, D.; Badolato, A.; Srinivasan, K., Efficient quantum dot single photon extraction into an optical fiber using a nanophotonic directional coupler. Appl. Phys. Lett. 2011, 99 (12), 121101.

12. Lin, X.; Dai, X.; Pu, C.; Deng, Y.; Niu, Y.; Tong, L.; Fang, W.; Jin, Y.; Peng, X., Electrically-driven single-photon sources based on colloidal quantum dots with near-optimal antibunching at room temperature. Nat. Commun. 2017, 8 (1), 1132.

13. Claudon, J.; Bleuse, J.; Malik, N. S.; Bazin, M.; Jaffrennou, P.; Gregersen, N.; Sauvan, C.; Lalanne, P.; Gérard, J.-M., A highly efficient single-photon source based on a quantum dot in a photonic nanowire. Nat. Photonics 2010, 4 (3), 174-177.

14. Versteegh, M. A. M.; Reimer, M. E.; Jöns, K. D.; Dalacu, D.; Poole, P. J.; Gulinatti, A.; Giudice, A.; Zwiller, V., Observation of strongly entangled photon pairs from a nanowire quantum dot. Nat. Commun. 2014, 5, 5298.

15. Deshpande, S.; Heo, J.; Das, A.; Bhattacharya, P., Electrically driven polarized singlephoton emission from an InGaN quantum dot in a GaN nanowire. Nat. Commun. 2013, 4, 1675.

16. Reimer, M. E.; Bulgarini, G.; Akopian, N.; Hocevar, M.; Bavinck, M. B.; Verheijen, M. A.; Bakkers, E. P.; Kouwenhoven, L. P.; Zwiller, V., Bright single-photon sources in bottom-up tailored nanowires. Nat. Commun. 2012, 3, 737.

17. Heiss, M.; Fontana, Y.; Gustafsson, A.; Wüst, G.; Magen, C.; O’regan, D.; Luo, J.; Ketterer, B.; Conesa-Boj, S.; Kuhlmann, A., Self-assembled quantum dots in a nanowire system for quantum photonics. Nat. Mater. 2013, 12 (5), 439.

18. Deshpande, S.; Bhattacharya, P., An electrically driven quantum dot-in-nanowire visible single photon source operating up to 150 K. Appl. Phys. Lett. 2013, 103 (24), 241117.

19. Tribu, A.; Sallen, G.; Aichele, T.; Andre, R.; Poizat, J.-P.; Bougerol, C.; Tatarenko, S.; Kheng, K., A high-temperature single-photon source from nanowire quantum dots. Nano Lett. 2008, 8 (12), 4326-4329.

20. Holmes, M. J.; Choi, K.; Kako, S.; Arita, M.; Arakawa, Y., Room-temperature triggered single photon emission from a III-nitride site-controlled nanowire quantum dot. Nano Lett. 2014, 14 (2), 982-6.

21. Haffouz, S.; Zeuner, K. D.; Dalacu, D.; Poole, P. J.; Lapointe, J.; Poitras, D.; Mnaymneh, K.; Wu, X.; Couillard, M.; Korkusinski, M.; Schöll, E.; Jöns, K. D.; Zwiller, V.; Williams, R. L., Bright Single InAsP Quantum Dots at Telecom Wavelengths in Position-Controlled InP Nanowires: The Role of the Photonic Waveguide. Nano Lett. 2018, 18 (5), 3047-3052.

22. Holmes, M. J.; Kako, S.; Choi, K.; Arita, M.; Arakawa, Y., Single Photons from a Hot Solid-State Emitter at 350 K. ACS Photonics 2016, 3 (4), 543-546. 
23. Zhang, L.; Teng, C.-H.; Hill, T. A.; Lee, L.-K.; Ku, P.-C.; Deng, H., Single photon emission from site-controlled InGaN/GaN quantum dots. Applied Physics Letters 2013, 103 (19), 192114.

24. Senellart, P.; Solomon, G.; White, A., High-performance semiconductor quantum-dot single-photon sources. Nat. Nanotechnol. 2017, 12, 1026.

25. Hours, J.; Varoutsis, S.; Gallart, M.; Bloch, J.; Robert-Philip, I.; Cavanna, A.; Abram, I.; Laruelle, F.; Gérard, J. M., Single photon emission from individual GaAs quantum dots. Appl. Phys. Lett. 2003, 82 (14), 2206-2208.

26. Bayer, M.; Forchel, A., Temperature dependence of the exciton homogeneous linewidth in In $0.60 \mathrm{Ga} 0.40$ As / GaAs self-assembled quantum dots. Phys. Rev. B 2002, 65 (2), 321-325.

27. Ying, Y.; Xiu - Ming, D.; Bin, W.; Guo - Wei, Z.; Xiang - Jun, S.; Li, W.; Dan, S.; Jian Xing, X.; Hai - Yan, W.; Hai - Qiao, N.; Bao - Quan, S.; Yuan, J.; Xiao - Dong, H.; Zhi - Chuan, N., Self - Assembled Quantum Dot Structures in a Hexagonal Nanowire for Quantum Photonics. Adv. Mater. 2014, 26 (17), 2710-2717.

28. Cavigli, L.; Bietti, S.; Accanto, N.; Minari, S.; Abbarchi, M.; Isella, G.; Frigeri, C.; Vinattieri, A.; Gurioli, M.; Sanguinetti, S., High temperature single photon emitter monolithically integrated on silicon. Appl. Phys. Lett. 2012, 100 (23), 231112.

29. Kiravittaya, S.; Benyoucef, M.; Zapf-Gottwick, R.; Rastelli, A.; Schmidt, O. G., Ordered GaAs quantum dot arrays on $\mathrm{GaAs}(001)$ : Single photon emission and fine structure splitting. Appl. Phys. Lett. 2006, 89 (23), 233102.

30. Wu, J.; Ramsay, A.; Sanchez, A.; Zhang, Y.; Kim, D.; Brossard, F.; Hu, X.; Benamara, M.; Ware, M. E.; Mazur, Y. I.; Salamo, G. J.; Aagesen, M.; Wang, Z.; Liu, H., Defect-Free SelfCatalyzed GaAs/GaAsP Nanowire Quantum Dots Grown on Silicon Substrate. Nano Lett. 2016, 16 (1), 504-511.

31. Yu, P.; Wu, J.; Liu, S.; Xiong, J.; Jagadish, C.; Wang, Z. M., Design and fabrication of silicon nanowires towards efficient solar cells. Nano Today 2016, 11 (6), 704-737.

32. Borgström, M. T.; Zwiller, V.; Müller, E.; Imamoglu, A., Optically Bright Quantum Dots in Single Nanowires. Nano Lett. 2005, 5 (7), 1439-1443.

33. Allen, J. E.; Hemesath, E. R.; Perea, D. E.; Lensch-Falk, J. L.; Li, Z.; Yin, F.; Gass, M. H.; Wang, P.; Bleloch, A. L.; Palmer, R. E., High-resolution detection of Au catalyst atoms in $\mathrm{Si}$ nanowires. Nat. Nanotechnol. 2008, 3 (3), 168.

34. Le Ru, E. C.; Fack, J.; Murray, R., Temperature and excitation density dependence of the photoluminescence from annealed InAs/GaAs quantum dots. Phys. Rev. B 2003, 67 (24), 245318. 35. Yeo, I.; Malik, N. S.; Munsch, M.; Dupuy, E.; Bleuse, J.; Niquet, Y.-M.; Gérard, J.-M.; Claudon, J.; Wagner, É.; Seidelin, S.; Auffèves, A.; Poizat, J.-P.; Nogues, G., Surface effects in a semiconductor photonic nanowire and spectral stability of an embedded single quantum dot. Appl. Phys. Lett. 2011, 99 (23), 233106.

36. Lu, D.; Zhang, Y.; Lai, M.; Lee, A.; Xie, C.; Lin, J.; Lei, T.; Lin, Z.; Kley, C. S.; Huang, J.; Rabani, E.; Yang, P., Giant Light-Emission Enhancement in Lead Halide Perovskites by Surface Oxygen Passivation. Nano Lett. 2018, 18 (11), 6967-6973.

37. Liu, J.; Konthasinghe, K.; Davanço, M.; Lawall, J.; Anant, V.; Verma, V.; Mirin, R.; Nam, S. W.; Song, J. D.; Ma, B., Single Self-Assembled InAs/GaAs Quantum Dots in Photonic Nanostructures: The Role of Nanofabrication. Phys. Rev. Appl. 2018, 9 (6), 064019.

38. Timm, R.; Eisele, H.; Lenz, A.; Becker, S. K.; Grabowski, J.; Kim, T.-Y.; Müller-Kirsch, L.; Pötschke, K.; Pohl, U. W.; Bimberg, D.; Dähne, M., Structure and intermixing of GaSb / GaAs quantum dots. Appl. Phys. Lett. 2004, 85 (24), 5890-5892.

39. Sun, M. H.; Joyce, H. J.; Gao, Q.; Tan, H. H.; Jagadish, C.; Ning, C. Z., Removal of Surface 
States and Recovery of Band-Edge Emission in InAs Nanowires through Surface Passivation. Nano Lett. 2012, 12 (7), 3378-3384.

40. Sandroff, C. J.; Hegde, M. S.; Farrow, L. A.; Chang, C. C.; Harbison, J. P., Electronic passivation of GaAs surfaces through the formation of arsenic - sulfur bonds. Appl. Phys. Lett. 1989, 54 (4), 362-364.

41. Joyce, H. J.; Parkinson, P.; Jiang, N.; Docherty, C. J.; Gao, Q.; Tan, H. H.; Jagadish, C.; Herz, L. M.; Johnston, M. B., Electron Mobilities Approaching Bulk Limits in "Surface-Free" GaAs Nanowires. Nano Lett. 2014, 14 (10), 5989-5994.

42. Friedler, I.; Sauvan, C.; Hugonin, J. P.; Lalanne, P.; Claudon, J.; Gérard, J. M., Solid-state single photon sources: the nanowire antenna. Opt. Express 2009, 17 (4), 2095-2110.

43. Bounouar, S.; Elouneg-Jamroz, M.; Hertog, M. d.; Morchutt, C.; Bellet-Amalric, E.; André, R.; Bougerol, C.; Genuist, Y.; Poizat, J. P.; Tatarenko, S.; Kheng, K., Ultrafast Room Temperature Single-Photon Source from Nanowire-Quantum Dots. Nano Lett. 2012, 12 (6), 2977-2981.

44. Wang, J.; Gudiksen, M. S.; Duan, X.; Cui, Y.; Lieber, C. M., Highly Polarized Photoluminescence and Photodetection from Single Indium Phosphide Nanowires. Science 2001, 293 (5534), 1455-1457.

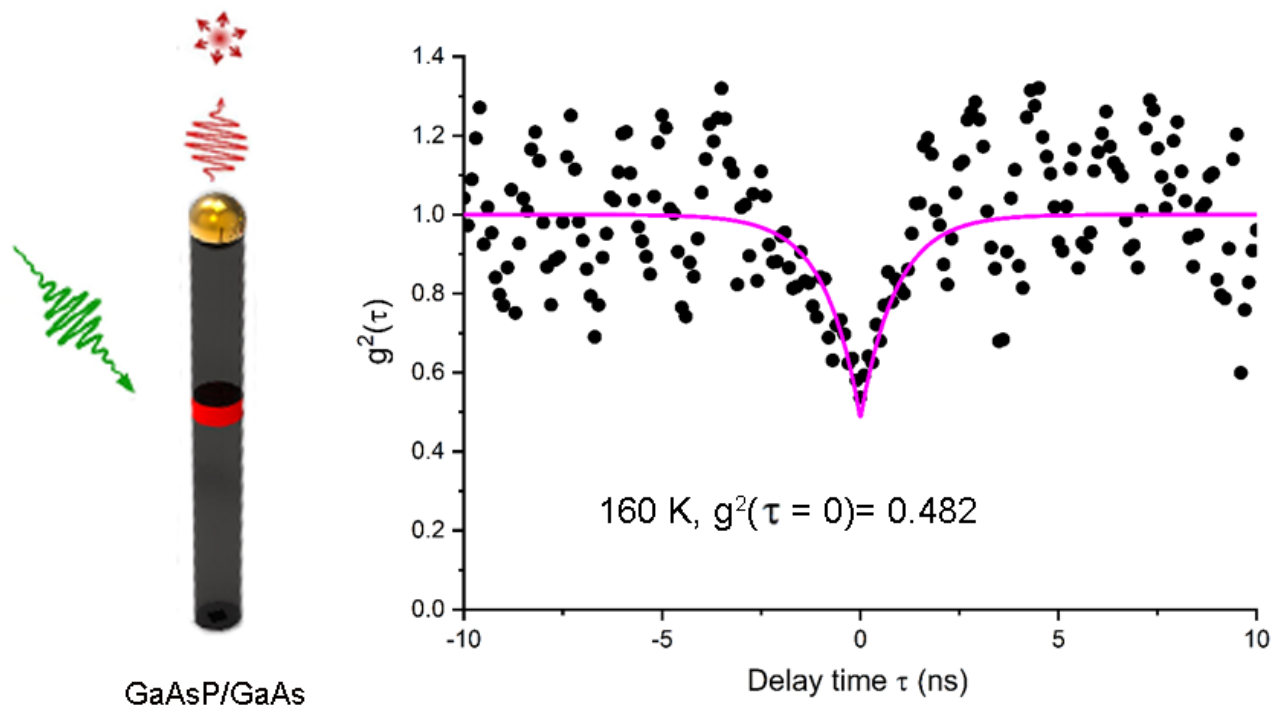

